\title{
Magnetoencephalography Cortical Source Imaging Using Spherical Mapping
}

\author{
Chang-Hwan $\mathrm{Im}^{1}$, Chany Lee ${ }^{1}$, Hyun-Kyo Jung ${ }^{1}$, Yong-Ho Lee ${ }^{2}$, and Shinya Kuriki ${ }^{3}$ \\ ${ }^{1}$ School of Electrical Engineering and Computer Science (SoEECS), Seoul National University, Seoul 151-744, Korea \\ ${ }^{2}$ Korea Research Institute of Standards and Science (KRISS), Daejeon 305-600, Korea \\ ${ }^{3}$ Research Institute for Electronic Science, Hokkaido University, Sapporo 060-0812, Japan
}

\begin{abstract}
This paper proposes a novel approach to enhancing results of magnetoencephalography cortical source imaging. The proposed approach utilizes bell-shaped functions defined on an inflated cortical surface, which has one-to-one correspondence with original tessellated cortical surface. The coefficients of the functions are then determined using sensitivity analysis with conjugate gradient updating scheme. Applications of the approach to a simulation study and a practical experiment have resulted in more stable and smoother brain source distribution, compared to conventional linear inverse approach.
\end{abstract}

Index Terms-Brain surface inflation, cortical source imaging, inverse problem, magnetoencephalography (MEG).

\section{INTRODUCTION}

$\mathbf{T}$ HE ABILITY to explore regional functions of a brain has attracted permanent interest of many neuroscientists. Among various brain mapping techniques, magnetoencephalography (MEG) is becoming an indispensable method in many clinical applications and neuroscience due to its superior temporal resolution [1], [2]. The main object of the MEG inverse problem is to reconstruct and localize the human brain electrical sources using magnetic field measurement outside the head. To localize the electric sources inside the brain, various source assumptions and reconstruction techniques have been proposed [2]. For example, equivalent current dipole (ECD), distributed source model, and scan methods such as multiple signal classification (MUSIC) and synthetic aperture magnetometry (SAM) have been proposed. Among them, the distributed source model with minimum-norm estimation (MNE) has been widely studied since Hämäläinen and Ilmoniemi [3], [4]. Their MNE selects the solution where the L2-norm of the current distribution was smallest. This fundamental study has led several modifications [5]. For example, low-resolution electrical tomography method (LORETA) gave deeper sources the same opportunity of being nicely reconstructed by the MNE [6]. Iterative focalization approaches such as FOCal Underdetermined System Solution (FOCUSS) were proposed to solve the underdetermined inverse problem more effectively and reconstruct more focalized solutions [7].

Recently, to reduce the dimension of the source space, anatomical constraints have been widely used. Dale and Sereno first proposed constraining the source space into anatomically known locations (interface between white and gray matter of the cerebral cortex) and orientations (perpendicular to the cortical surface), and weighting the estimate based on a priori information [8]. To use the anatomical constraints, cortical surface was extracted from MRI data and tessellated using numerous triangular elements [9]. After their work, most recent

Digital Object Identifier 10.1109/TMAG.2005.846286 publications on the distributed source model have adopted the anatomical constraints [10], [11]. The anatomically constrained distributed source model is usually called a cortically distributed source model or cortical source imaging. The use of anatomical constraints considerably enhanced robustness and accuracy of the solution, compared to the conventional volume pixel (voxel)-based reconstruction.

However, the cortical source imaging still has some problems to be solved as follows. 1) Because neuronal current flow generally generates very small magnetic field, about several tens of fT, considerable noise components are added to measured data. To stabilize the solution, one should introduce some special regularization techniques, which is still a problem being studied actively [5], [8], [12]. 2) The cortical source imaging suffers from unwanted spurious sources or many local peaks due to the highly underdetermined relationship between the number of unknowns (point sources defined on tessellated cortical surface; over several thousand) and that of measured data (less than a few hundred) [10]. 3) If we regard that the tessellated cortical surface is sufficiently accurate, constraining the orientations of scattered sources as well as the positions of them is physiologically plausible since actual generators of MEG signals are arranged perpendicularly to the cortical surface. However, the authors found that the orientation constraints usually made the distributions of cortical sources to be discontinuous, especially around gyri. The main reason for the problem is that radial sources (normal direction to conductor surface) located on gyri generate smaller magnetic field than tangential ones on sulci [1]. The discontinuous source distribution makes it difficult for one to estimate and characterize actual brain activations.

In this paper, a novel approach to enhance reconstructed source images is proposed. We first inflated tessellated cortical surface into a sphere [9], and then assumed a spatial function defined on the sphere. The function was defined as the summation of several bell-shaped functions, which was once used for electrical impedance tomography (EIT) in two-dimensional space [13]. The function represents spatial distribution of source strength. Because the vertices on the tessellated cortical surface have one-to-one correspondence with those on the inflated sphere, we could express brain activations on the actual cortical surface using the spherical function. The parameters of the 


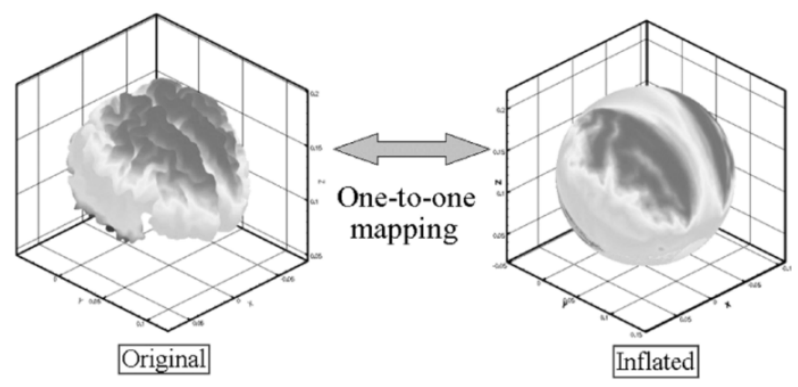

Fig. 1. Concept of one-to-one correspondence characteristic between original and inflated cortical surface meshes. Colors represent node numbers.

spherical function were determined using sensitivity analysis with conjugate gradient (CG) updating scheme [14]. The value of an error function was calculated in the real source space; while that of source distribution was estimated in the spherical domain. In this paper, we applied the proposed approach to a simulation study and a practical experiment, and found that most of local peaks and discontinuities were eliminated and smoother images could be obtained, compared to linear inverse approaches.

\section{METHODS}

\section{A. Conventional Approach-Linear Inverse Estimation}

For comparison, we applied a linear estimation approach [8], [11] to reconstruct distributed brain electrical sources. The expression for the inverse operator $\mathbf{W}$ is

$$
\mathbf{W}=\mathbf{R A}^{\mathrm{T}}\left(\mathbf{A R} \mathbf{A}^{\mathrm{T}}+\mathbf{C}\right)^{-1}
$$

where $\mathbf{A}$ is the lead field matrix that relates point sources to sensors [1], $\mathbf{R}$ is a source covariance matrix, and $\mathbf{C}$ is a noise covariance matrix. The source distribution can be estimated by multiplying $\mathbf{W}$ by the measured signal at a specific instant. If we assume that both $\mathbf{R}$ and $\mathbf{C}$ are scalar multiples of identity matrix, this approach becomes identical to minimum norm estimation [11]. In our study, we assumed the source covariance matrix $\mathbf{R}$ to be a diagonal matrix, which means that we ignored cross relationships between neighboring sources [11]. The noise covariance matrix $\mathbf{C}$ was also set to be a diagonal matrix under the assumption that common noise components were eliminated during signal processing.

\section{B. One-to-One Correspondence Characteristic}

A brain cortical surface was extracted from MRI T1 image $(256 \times 256 \times 200$, voxel size for each direction: $1 \mathrm{~mm})$ and tessellated into about 350000 triangular elements including about 170000 vertices. To extract and tessellate the cortical surface, we applied BrainSuite, developed at the University of Southern California, CA [15]. The cortical surface was also inflated into a sphere using the same software. During the inflation, total number of nodes and information on mesh connectivity were preserved. Fig. 1 shows the corresponding node information between original cortical surface and inflated sphere. Colors in the figures represent node numbers. We can see from the figures that all the vertices included in the original cortical surface can be mapped onto the corresponding vertices on the inflated sphere and can have their own spherical coordinates (angles). Generally, the inflated cortical surface has been used for visualization

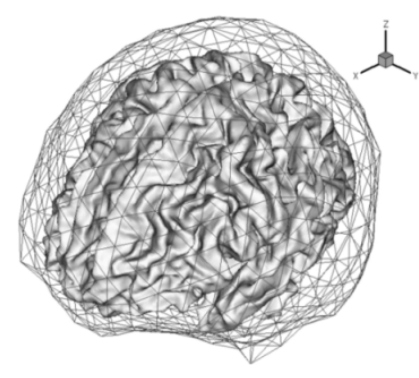

Fig. 2. Boundary element meshes (inner skull boundary). Note that the cortical surface was not included in the boundary element analysis. The cortical surface tessellation was used only for locating dipolar sources.

purpose in order to present the source distribution more intuitively [9]. In this paper, however, it will be directly used for the brain source reconstruction, for the first time.

\section{Proposed Approach}

A function used for the spherical mapping was defined as the summation of the following bell-shaped functions:

$$
f_{i}=\frac{b_{i}}{1+\left(\frac{\theta}{a_{i}}\right)^{2}}
$$

where $\theta$ is an angle between peak of the single function and an evaluating point. To evaluate the bell-shaped function, four parameters should be determined: $a_{i}, b_{i}$, and two angular coordinates of the peak position, $\theta_{P, i}$ and $\varphi_{P, i}$. In this paper, the single function represents one brain activation around the peak position, $\theta_{P, i}$ and $\varphi_{P, i}$. The value of $b_{i}$ determines the magnitude of the activation; whereas that of $a_{i}$ determines range of the activation. Because all vertices on a tessellated cortical surface have one-to-one correspondence with those on the inflated sphere, the current source intensity at each vertex of original cortical surface can be evaluated by substituting a coordinate of the corresponding sphere vertex for $\theta$. Then, magnetic field at each sensor can be calculated using the evaluated source intensity. On the other hand, the objective function to be minimized was defined as

$$
E=\left(\mathbf{B}_{\mathbf{m}}-\mathbf{B}_{\mathbf{c}}\right)^{T}\left(\mathbf{B}_{\mathbf{m}}-\mathbf{B}_{\mathbf{c}}\right)
$$

where $\mathbf{B}_{\mathbf{m}}$ is a measured magnetic field vector at sensors and $\mathbf{B}_{\mathbf{c}}$ is a calculated magnetic field vector. For the calculation of the magnetic field, we applied boundary element method (BEM) [1], [2]. It has been frequently reported that just considering inner skull boundary is sufficient for the MEG calculations [16], [17]. The boundary surface was composed of 1016 elements and 510 nodes. Fig. 2 shows the boundary element meshes and the tessellated cortical surface.

To evaluate parameters of the bell-shaped function, the gradient of the objective function was calculated. Because it is hard to relate the spherical function and calculated magnetic field using a simple mathematical expression, the gradients $\left(d E / d a_{i}\right.$, $d E / d b_{i}, d E / d \theta_{P, i}$, and $\left.d E / d \varphi_{P, i}\right)$ were evaluated using numerical differentiation.

To update the parameters, a conjugated gradient (CG) updating scheme was applied. During the iteration, the maximum value of $a_{i}$ was restricted to prevent an activating region from expending too much. In this study, we set the value to be 


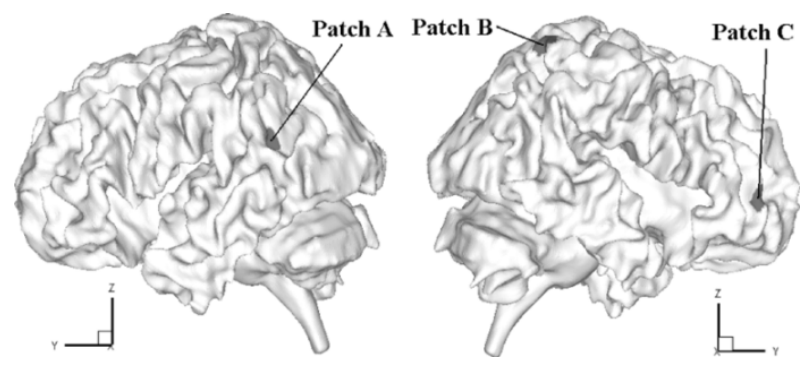

Fig. 3. Locations of three cortical patches to simulate realistic MEG data.

$\pi / 45(\mathrm{rad})$ from our experiences. Initial value of $b_{i}$ should be assumed to be a very small value, not zero, because initial gradients of other parameters needed to be calculated. In this study, the CG iteration stopped when the total sum of the gradients decreased below a predetermined value ( $1 \%$ of the initial value). After the updating processes, some small activations below a noise level were eliminated from final distribution.

\section{A Simulation StUdy USING Forward DATA}

The inverse technique introduced in previous chapter was applied to artificially constructed forward data. We assumed realistic conditions obtained from a practical measurement that will be used again in the next chapter. The sensor layout used for the simulation was a 204-channel whole-head SQUID gradiometer system (http://www.neuromag.com).

For the forward calculation, we placed three cortical activation patches, of which the locations are shown in Fig. 3. The cortical patches were generated using the following process. 1) A point is selected as a seed of an activation patch area. 2) The patch area is extended by including neighboring vertices around the patch. 3) If the total area of the cortical patch exceeds an aimed surface area, the extension of the activation patch is terminated. The source intensity patterns $I$ given to vertices inside the patches were defined as follows:

Patch A:

$$
\begin{aligned}
I & =-h \times 10^{-4}(t-100)^{2}+h & & (0 \mathrm{~ms} \quad<t<200 \mathrm{~ms}) \\
& =0 & & (200 \mathrm{~ms}<t<400 \mathrm{~ms})
\end{aligned}
$$

Patches B and C:

$$
\begin{aligned}
I & =0 & & (0 \mathrm{~ms} \quad<t<200 \mathrm{~ms}) \\
& =-h \times 10^{-4}(t-300)^{2}+h & & (200 \mathrm{~ms}<t<400 \mathrm{~ms}) .
\end{aligned}
$$

where the value of $h$ was determined by means of a trial-anderror process in order to make the calculated magnetic field similar to the measurement data. After the forward calculation of magnetic field assuming $600-\mathrm{Hz}$ sampling rate, we added real brain noise to each sensor. The brain noise was obtained from prestimulus period of a practical experiment and scaled in order for signal-to-noise ratio to be approximately 10. Fig. 4 shows the finally constructed signal patterns for 204 channels with respect to simulated time.

We first reconstructed source distributions at times of 100 and $300 \mathrm{~ms}$ using conventional linear inverse estimation. For imposing an anatomical constraint in linear inverse estimation, the tessellated cortical surface was sampled to be about 10000 dipole locations. Fig. 5 shows the source distributions estimated at 100 and $300 \mathrm{~ms}$ using the linear inverse estimation. In the reconstructed images, many local peaks and discontinuity

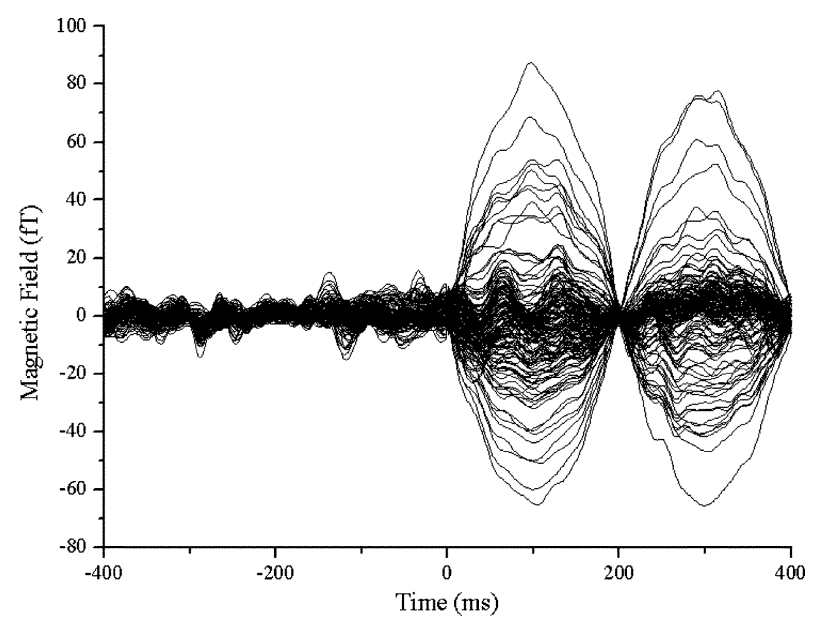

Fig. 4. Simulated MEG signals. Real brain noise $(\mathrm{SNR}=10)$ was added to each sensor.
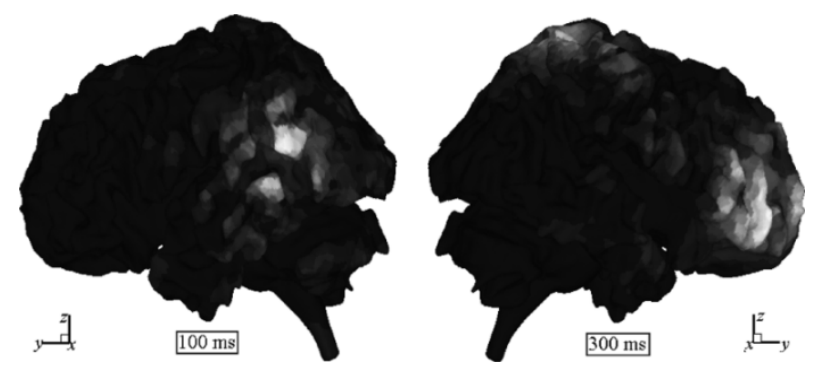

Fig. 5. Source distributions reconstructed by linear inverse estimation at $100 \mathrm{~ms}$ (left) and $300 \mathrm{~ms}$ (right). Brighter color represents higher intensity.

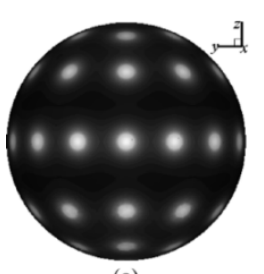

(a)

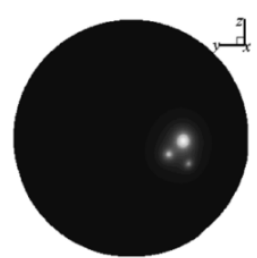

(b)

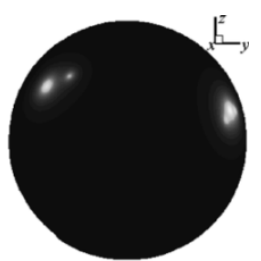

(c)
Fig. 6. Distributions of the bell-shaped function: (a) at initial stage, (b) at $100 \mathrm{~ms}$, (c) at $300 \mathrm{~ms}$. Brighter color represents higher intensity.
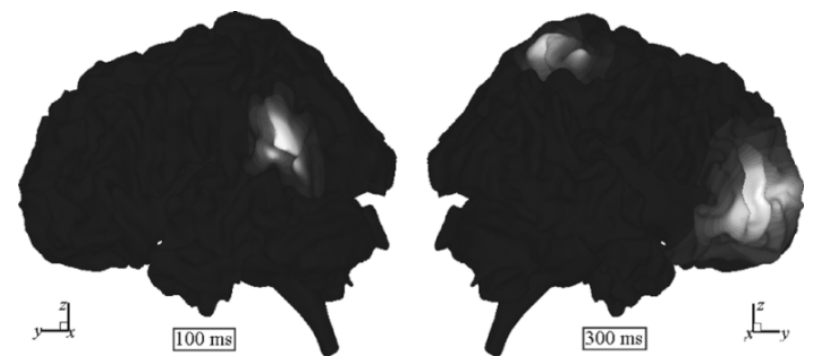

Fig. 7. Source distributions reconstructed by spherical mapping approach at $100 \mathrm{~ms}$ (left) and $300 \mathrm{~ms}$ (right). Brighter color represents higher intensity.

along the gyri are observed as expected. Then, we applied the proposed spherical mapping approach at the same time slices. Fig. 6(a)-(c) shows the distributions of the bell-shaped function at initial stage (49 peaks to make overdetermined relationship), at $100 \mathrm{~ms}$, and at $300 \mathrm{~ms}$, respectively. Fig. 7 shows the resultant distributions of sources mapped on the real cortical surface. 


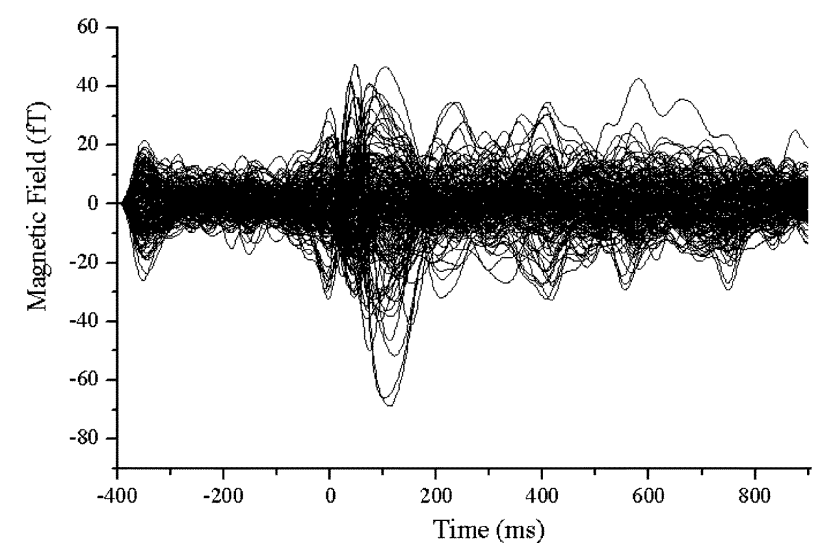

Fig. 8. MEG waveform averaged over 90 trials and filtered to $0.3-20 \mathrm{~Hz}$.
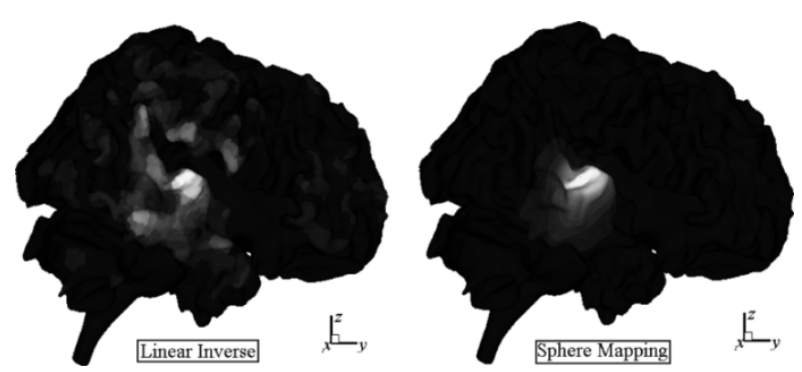

Fig. 9. Source distributions reconstructed by linear inverse estimation (left) and spherical mapping approach (right). Brighter color represents higher intensity.

It can be easily seen from the results that the spherical mapping approach yielded smoother images along the cortical surface and eliminated unnecessary ripples included in the conventional results, which will make it easier to identify and characterize estimated source distribution.

\section{ApPliCATION to A PRACTICAL EXPERIMENT}

The proposed approach was applied to a simple MEG experiment. Auditory evoked field (AEF) was measured using the 204-channel MEG system introduced in the previous simulation, after stimulating a pure tone sound to a subject's left ear. Fig. 8 shows the measured MEG waveform. It is a very well known fact that an auditory evoked response at $100 \mathrm{~ms}$ (N100m) usually appears around Sylvain fissure in temporal lobe of the opposite hemisphere [18]. Fig. 9 shows the source distributions reconstructed by the linear inverse estimation and the spherical mapping approach. From the results, the same conclusions could be made as in the previous simulation.

\section{CONCLUSION}

In this paper, a new approach to enhance reconstructed brain source images using spherical function approximation was proposed. The approach utilized inflated cortical surface, which has been used only for visualization purpose, to reconstruct smoother source images on highly curved cortical surfaces. Its applications to simulation and experimental studies showed improved source distributions, which were easier to identify and characterize, compared to a conventional linear inverse estimation. Further studies should be continued to reduce increased computational cost, which stems from nonlinear optimization processes used for the approach.

\section{ACKNOWLEDGMENT}

This work was supported in part by the NRL Project of the Ministry of Science and Technology, Korea.

\section{REFERENCES}

[1] M. S. Hämäläinen, R. Hari, R. J. Ilmoniemi, J. Knuutila, and O. V. Lounasmaa, "Magnetoencephalography. Theory, instrumentation and applications to the noninvasive study of human brain function," Rev. Mod. Phys., vol. 65, pp. 413-497, 1993.

[2] S. Baillet, J. C. Mosher, and R. M. Leahy, "Electromagnetic brain mapping," IEEE Signal Process. Mag., pp. 14-30, Nov. 2001.

[3] M. S. Hämäläinen and R. J. Ilmoniemi, "Interpreting measured magnetic fields of the brain: estimates of current distributions," Helsinki Univ. Technol., Tech. Rep. TKK-F-A559, 1984.

[4] - "Interpreting magnetic fields of the brain: minimum norm estimates," Med. Biol. Eng. Comput., vol. 32, pp. 35-42, 1994.

[5] S. Baillet, J. J. Riera, G. Marin, J. F. Mangin, J. Aubert, and L. Garnero, "Evaluation of inverse methods and head models for EEG source localization using a human skull phantom," Phys. Med. Biol., vol. 46, pp. 77-96, 2001.

[6] R. M. Pascual-Marqui, C. Michel, and D. Lehman, "Low resolution electromagnetic tomography: a new method for localizing electrical activity in the brain," Int. J. Psychophysiol., vol. 18, pp. 49-65, 1994.

[7] I. F. Gorodnistky, J. S. George, and B. D. Rao, "Neuromagnetic imaging with FOCUSS: a recursive weighted minimum norm algorithm," Electroencephalogr. Clin. Neurophysiol., vol. 95, pp. 231-251, 1995.

[8] A. M. Dale and M. Sereno, "Improved localization of cortical activity by combining EEG and MEG with MRI surface reconstruction: a linear approach," J. Cognit. Neurosci., vol. 5, pp. 162-176, 1993.

[9] A. M. Dale, B. Fischl, and M. I. Sereno, "Cortical surface-based analysis-I. Segmentation and surface reconstruction," Neuroimage, vol. 9, pp. 179-194, 1999.

[10] C.-H. Im, K.-O. An, H.-K. Jung, H. Kwon, and Y.-H. Lee, “Assessment criteria for MEG/EEG cortical patch tests," Phys. Med. Biol., vol. 48, pp. 2561-2573, 2003.

[11] A. K. Liu, A. M. Dale, and J. W. Belliveau, "Monte Carlo simulation studies of EEG and MEG localization accuracy," Hum. Brain Map., vol. 16, pp. 47-62, 2002.

[12] J. Schreiber, J. Haueisen, and J. Nenonen, "A new method for choosing the regularization parameter in time-dependent inverse problems and its application to magnetocardiography," IEEE Trans. Magn., vol. 40, no. 2, pp. 1104-1107, Mar. 2004.

[13] R. Sikora, Z. Giza, F. Filipowicz, and J. Sikora, "The Bell function approximation of material coefficients distribution in the electrical impedance tomography," IEEE Trans. Magn., vol. 36, no. 4, pp. 1023-1026, Jul. 2000.

[14] C.-H. Im, H.-K. Jung, H. Kwon, and Y.-H. Lee, "Multiresolutive reconstruction of magnetoencephalography source distribution," IEEE Trans. Magn., vol. 40, no. 2, pp. 1100-1103, Mar. 2004.

[15] D. W. Shattuck and R. M. Leahy, "BrainSuite: an automated cortical surface identification tool," Med. Image. Anal., vol. 6, pp. 129-142, 2002.

[16] M. S. Hämäläinen and J. Sarvas, "Realistic conductivity geometry model of the human head for interpretation of neuromagnetic data," IEEE Trans. Biomed. Eng., vol. 36, no. 2, pp. 165-171, Feb. 1989.

[17] J. W. Meijs et al., "Relative influence of model assumptions and measurement procedures in the analysis of the MEG," Med. Biol. Eng. Comput., vol. 26, pp. 136-142, 1988.

[18] H. Kwon, Y.-H. Lee, J.-M. Kim, Y.-K. Park, and S. Kuriki, "Localization accuracy of single current dipoles from tangential components of auditory evoked fields," Phys. Med. Biol., vol. 47, pp. 4145-4154, 2002.

Manuscript received June 8, 2004. 